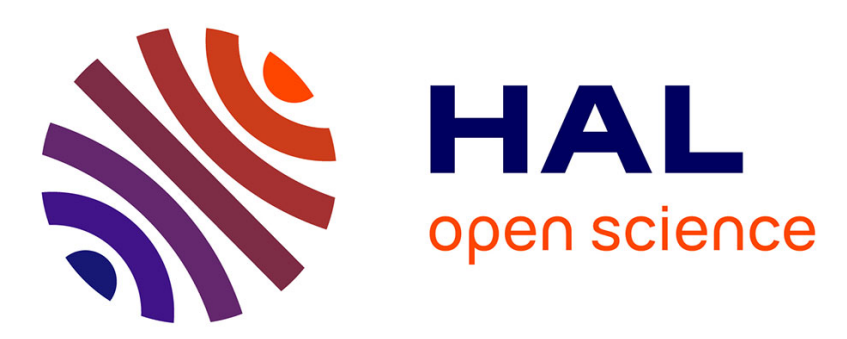

\title{
A Co-design Distributed Bayesian Approach for Decision and Scheduling of WNCS
}

Amine Mechraoui, Faris Djohor, Jean-Marc Thiriet, Sylviane Gentil

\section{To cite this version:}

Amine Mechraoui, Faris Djohor, Jean-Marc Thiriet, Sylviane Gentil. A Co-design Distributed Bayesian Approach for Decision and Scheduling of WNCS. IFAC WC 2011 - 18th IFAC World Congress, Aug 2011, Milan, Italy. pp.14970-14975. hal-00618954

\section{HAL Id: hal-00618954 https://hal.science/hal-00618954}

Submitted on 4 Sep 2011

HAL is a multi-disciplinary open access archive for the deposit and dissemination of scientific research documents, whether they are published or not. The documents may come from teaching and research institutions in France or abroad, or from public or private research centers.
L'archive ouverte pluridisciplinaire HAL, est destinée au dépôt et à la diffusion de documents scientifiques de niveau recherche, publiés ou non, émanant des établissements d'enseignement et de recherche français ou étrangers, des laboratoires publics ou privés. 


\title{
A Co-design Distributed Bayesian Approach for Decision and Scheduling of WNCS
}

\author{
A. Mechraoui * F. Djohor ${ }^{* *}$ J-M. Thiriet* S. Gentil* \\ * Department of Control Systems Engineering, GIPSA-lab, University \\ of Grenoble, 38402, France ; e-mail: amine.mechraoui, \\ jean-marc.thiriet, sylviane.gentil@gipsa-lab.inpg.fr). \\ ** Laboratoire des Sciences de l'Information et des Systèmes, LSIS, \\ Domaine universitaire de Saint Jérôme 13397 Marseille, France ; \\ e-mail: faris.djohor@lsis.org.
}

\begin{abstract}
This paper deals with the problem of co-design of Wireless Networked Control systems (WNCS). The integration of the Wireless Network (WN) in the control loop influences the Quality of Control (QoC) of the system in terms of the Quality of Service (QoS) of the WN. We present a co-design approach for WNCS based on distributed Bayesian Network (BN) and we illustrate it with Khepera III robot. The proposed BN is constructed from the robot experimental data. This approach allows to make decisions to ensure a good QoC for the robot and also ensure that the QoS is always sufficient to maintain a good QoC. Keeping a sufficient QoS depends on the scheduling method used and therefore the proposed approach aims also to determine the proper scheduling method to keep the system's performance. Finally a comparison is performed with the classical time-triggered scheduling. The results obtained with BN scheduling are better in terms of QoS and QoC for WNCS.
\end{abstract}

Keywords: Wireless Networked Control Systems, Co-design, Bayesian Network, Scheduling, Decision-making, Mobile robot.

\section{INTRODUCTION}

Wireless Networked Control Systems (WNCS) are distributed systems in which the information between the node controller and node system are exchanged via a wireless communication network. Compared with traditionally known controlled systems, the Quality of control (QoC) in the WNCS depends mainly on the Quality of Service (QoS) of the used Wireless Network (WN). Using a WN to interconnect different nodes of a distributed system brings many benefits. One of the advantages of WNCS is to reduce complexity in terms of installation. The weight and the limited space of WNCS may be one of the criteria for selecting such systems. The simplicity of maintenance also proves a great advantage for these systems. These benefits can also increase system flexibility. Therefore, the WNCS have found applications in various fields such as mobile sensor networks [Leonard et al., 2007], robotics and mobile robotics [Arzen et al., 2007, Cremean et al., 2006], Unmanned Aerial Vehicles UAV [Berbra et al., 2009] etc. . However, the integration of the WN in the control loop introduces some difficult problems to solve.

Traditionally known Controlled Systems are based on the assumption that all transmitted information is received without loss and delay, which means that the link between the various components of the system is considered as "perfect". However, in Networked Control Systems (NCS), the network either wired or wireless is "imperfect", and the modeling and analysis of such NCS are more complex
[Hespanha et al., 2007]. The network influence on the QoC of the system can be due to sampling, delay, packets loss and the rate of the WN.

A lot of works have been done recently on these topics. According to various aspects of the analysis and design of the NCS, existing works can be broadly divided into four categories: stability analysis [Hespanha et al., 2007, Liu et al., 2008, Xiaomei et al., 2008], state estimation [Giorgiev and Tilbury, 2006, Sinopoli et al., 2004], control synthesis [Varsakelis and Zhang, 2008, Guo and Li, 2010, Fu and Dai, 2009] and co-design of control and communication [Aubrun et al., 2010, Branicky et al., 2002, 2003]. Co-design approach is a new research domain. In such approach, problems due to the network such as delays, packet loss and jitter (QoS) are considered simultaneously with the control problems such as stability and performance of the controller (QoC). Generally, NCS has to be assigned to a schedule of transmission for each transmission entity (sensor, controller) based on a scheduling algorithm. Usually, the control system design and network scheduling are studied separately. This separation allowed the automatic community to focus on this particular field without taking into account the scheduling. But with the growing use of NCS, the need to address simultaneously the problem of scheduling and control encouraged the researchers to work on a so-called problem of co-design (adapt the network to the dynamic needs of the control and vice-versa) Fig.1. Therefore, understanding the impact of scheduling on the stability and performance of the control 
has become crucial. Indeed, recent years have allowed the automatic community to study the communication network [Branicky et al., 2002, Cervin and Eker, 2005, Branicky et al., 2003]. All these works are focused on NCS and less works have been done till now on WNCS. This paper proposes a novel approach of Co-design of WNCS.

The causality between the different parameters of the WN and the QoS and the QoC permits to use the graph theory to model the system. The probability theory can also be used according to the stochastic aspect of WN. Hence, we propose in this paper to use Bayesian Network (BN) [Pearl, 1988], [Naim et al., 2004] to model the QoS and the QoC of the WNCS as it brings graph theory and probability theory together. $\mathrm{BN}$ is used in this paper to schedule the information and to make on-line decision to avoid both degradation of the QoS and the QoC. The reconfiguration of the WN is required to fulfill the need of control in the case of degradation of the QoC. The adaptation of control is required too in the case of the degradation of the QoS of the WN.

To illustrate our proposals, the communication architecture for two mobile robots moving with obstacle avoidance strategy in a $2 \mathrm{D}$ space with fixed control station is considered. According to the information delivered by the proposed $\mathrm{BN}$, the reconfiguration of both the communication architecture (Dynamic priority) and the strategy of control is possible by using different methods of tasks scheduling.

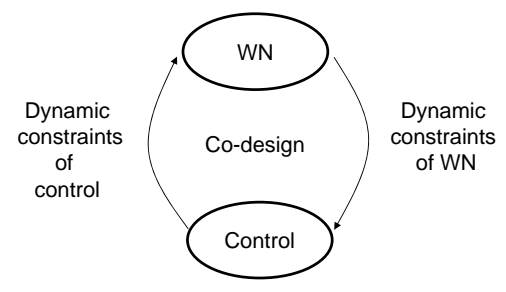

\section{Fig. 1. Co-design problem}

The paper is organized as follows. Section 2 presents the co-design problem and describes the proposed BN approach. The qualitative and the quantitative approach to model the proposed distributed BN using the experimental results are presented. The decision and scheduling approach using distributed BN are discussed in section 3 . Section 4 demonstrates the effectiveness of the approach using simulated results for two robots and two scenario are studied and compared. Section 5 gives conclusion and some perspectives.

\section{CO-DESIGN PROBLEM}

Distributed systems generally incorporate communication resources (such as networks and wireless networks) to connect their different subsystems. This category of systems requires appropriate scheduling taking into account stochastic phenomena and decision to ensure the reconfiguration of the communication resource to maintain an acceptable QoC for the system, and also to ensure the adaptation of the control strategy to maintain a good QoS of the network. To solve this co-design problem, we need also to locate on-line the source of degradation of the QoC and QoS. We propose Bayesian networks to come through these tasks and to achieve scheduling and decision making of the system.

The system considered in this paper is KHEPERA III mobile robot. The modeling methodology of the robot and the strategy of communication of two robots is presented in [Mechraoui et al., 2010, 2009]. For this robot, two missions are described (reach a target and avoid obstacles).

\subsection{Definition of Bayesian network}

Formally, BNs are directed acyclic graphs whose nodes represent random variables in the Bayesian meaning: they may be observable quantities, latent variables, unknown parameters or hypotheses. Edges represent causal relationships; nodes which are not connected represent variables which are conditionally independent from each other. Each node is associated with a probability function that takes as input a particular set of values for the node's parent variables and gives the probability of the variable represented by the node. Efficient algorithms exist that perform inference and learning (structure and parameter) in $\mathrm{BN}$ (see [Naim et al., 2004] for more details).

Many definition of BN exist in the literature. For what follows, let $G=(V, E)$ be a directed acyclic graph, and let $x=\left(x_{v}\right)_{v \in V}$ be a set of random variables indexed by $V$. $x$ is a BN with respect to $\mathrm{G}$ if its joint probability density function can be written as a product of the individual density function, conditional on their parent variables:

$$
p(x)=\prod_{v \in V} p\left(x_{v} \mid x_{p a(v)}\right)
$$

Where $p a(v)$ is the set of parents of $v$.

\subsection{Distributed Bayesian Networks}

The modeling of a system using Bayesian networks (BN) takes two important steps: qualitative approach and quantitative approach. The first consists of determining the structure of the BN (i.e. causal relationships between nodes), the second consists of finding for each node $X$, the probability conditional distribution upon $X$ 's parents. In the simplest case, a BN can be specified by an expert and is then used to perform inference. In other applications defining the network is too complex for humans. In this case, the structure and the parameters of the local distributions must be learned from data (see [Naim et al., 2004] for more details). However, in distributed systems, nodes might not be physically connected. In our application, which consists of controlling a group of robots via a $\mathrm{WN}$, the idea of distributing the BN comes out from the fact that some of the nodes are aboard the robots, some are in the control station. In addition to that, the complexity of a $\mathrm{BN}$ render impossible embedding all of it in a robot, due to memory and energy consumption constraints [Mechraoui et al., 2009]. That led us to imagine a distribution strategy that can handle those constraints, and simplify as possible the complexity of the BN.

For our application, the structural modeling can be done, based on graph theory, by analyzing influence between the nodes. On the other hand, the quantitative approach will be performed by parameter learning of the BN, due to it's 
relative complexity, using BayesiaLab.

Table 1. Signification of different nodes of the $\mathrm{BN}$

\begin{tabular}{lr} 
Nodes & Signification \\
\hline QoC & Quality of Control \\
QoS & Quality of Service \\
$\mathrm{R}$ & State of the robot \\
$\mathrm{PL}$ & Packet loss \\
RSSI & Request Signal \\
$\Delta_{t}$ & Strength Indicator \\
Obst & Delay \\
D & Obstacle \\
Col & Distance between the station and the robot \\
$\mathrm{N}$ & Collision \\
$\mathrm{M}$ & Number of communicating nodes \\
& Mission \\
\hline
\end{tabular}

Qualitative approach The QoC is mainly influenced by three parameters: the network's QoS, the mission the robot $M$ and the state of the robot $R$. Referring to [Hespanha et al., 2007] and [Li and Wang, 2008], the QoS is influenced by the delay $\Delta_{t}$ between transmission and reception, and by the rate of packet loss $P L$. The signal strength represented by the Request Signal Strength Indicator $(R S S I)$ influences the packet loss. The $R S S I$ is affected directly by the distance $D$ and obstacles Obst between the robot and the control station. The delay $\Delta_{t}$ is influenced by the probability of collisions $\mathrm{Col}$ between sources (robots or other applications) sending information via the network. The node $\mathrm{Col}$ is affected by the number $N$ of robots communicating with the control station (see Fig.2, Tab.2 and Tab.1).

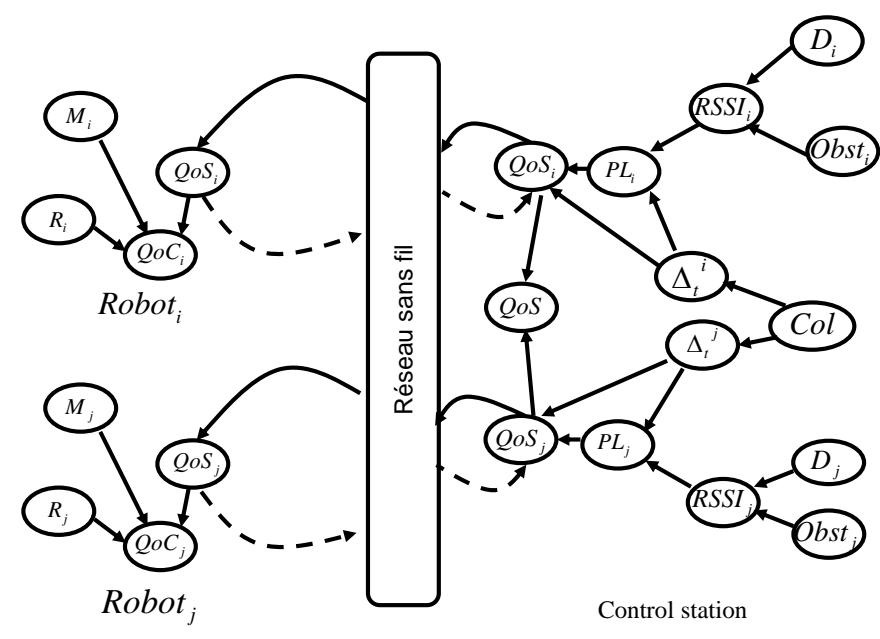

Fig. 2. Distributed Bayesian network

Quantitative approach Here we're looking for finding the probability distributions based on available data from a real robot. Two simple methods will be described here to perform this task. When all the variables are observable the simplest method is the statistical estimation which consists of estimating the probability of an event by the frequency of appearance of this event in a database constructed from series of tests on the real system. The estimator is then given by:

$$
\hat{P}\left(X_{i}=x_{k} \mid p a\left(X_{i}\right)=x_{j}\right)=\hat{\theta}_{i, j, k}^{L H}=\frac{N_{i, j, k}}{\sum_{k} N_{i, j, k}}
$$

Table 2. Node modes of BN

\begin{tabular}{|c|c|}
\hline Nodes & Modes \\
\hline QoC & Good, Degraded, Bad \\
\hline QoS & Good, Degraded, Bad \\
\hline $\mathrm{R}$ & Good, Degraded, Faulty \\
\hline PL & $\begin{aligned} 0 \% & \leq P L<24 \%, 24 \% \leq P L<46 \% \\
46 \% & \leq P L<74 \%, 74 \% \leq P L<100 \%\end{aligned}$ \\
\hline RSSI & $(\leq-48,>-40,-48<R S S \bar{I} \leq-40) d B m$ \\
\hline $\begin{array}{l}\Delta_{t} \\
\text { Obst }\end{array}$ & 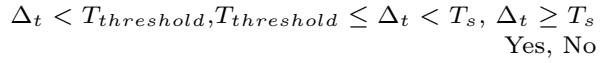 \\
\hline $\mathrm{D}$ & $D<D_{1}, D_{1} \leq D<D_{2}, D \geq D_{2}$ \\
\hline $\mathrm{Col}$ & yes, No \\
\hline $\mathrm{N}$ & Number of nodes \\
\hline M & Reach target, Obstacle avoidance \\
\hline
\end{tabular}

Where $N_{i, j, k}$ is the number of events in database for which the variable $X_{i}$ is in the state $x_{k}$ and its parents are in the configuration $x_{j}$. The statistical learning is chosen here over other methods for its simplicity. Database is constructed from series of tests on the real system and was obtained based on two criteria for such systems (robot unicycle and a $\mathrm{WN}$ ). These criteria are proposed to quantify the QoS and the QoC.

As stated above, the QoS is particularly influenced by packet loss and also the delay. To construct the database, we need to quantify the QoS for this system, and then qualify by ranges of values to define the modes (or states) of QoS. For this, we propose the following criterion:

$$
Q o S=\max \left(\frac{(1-P L) *\left(n T_{s}-\Delta_{t}\right)}{n T_{s}}, 0\right)
$$

$P L$ is the probability of packet loss, it is defined by tests on the real system. $\Delta_{T}$ is the delay between the sensor and the controller. $T_{s}$ is the sampling period. $n$ is a constant that characterizes the maximum number of lost samples that can be tolerated in which the system works perfectly. the QoC is influenced mainly by three parameters, namely QoS, the state of the robot and the system's mission. This leads us to propose the following criterion:

$$
Q \circ C=Q \circ S * \kappa_{j} * \varrho_{i}
$$

$\kappa_{j}$ is a variable that depends on the state $j$ of the robot such that $j \in\{$ Good, degraded or bad $\}$ and $\kappa_{\text {good }}>\kappa_{\text {deg }}>$ $\kappa_{\text {bad }}$, in our case we took: $\kappa_{\text {good }}=1, \kappa_{\text {deg }}=0.5$ and $\kappa_{b a d}=0 . \varrho_{i}$ is a variable that depends on the mission $i$ of the robot $(i \in\{$ obstacle, target $\})$ as $\varrho_{\text {obstacle }}<\varrho_{\text {target }}$ $\left(\varrho_{\text {target }}=1\right.$ and $\left.\varrho_{\text {obstacle }}=0.5\right)$. Fig.3 shows the variation of the QoC depending on the QoS in two different cases ("Reach a target" and "Avoid obstacle"). We note that the robot needs more QoS in the case of obstacle avoidance. The decreasing of the QoS is caused by the number of nodes that communicates.

\section{DISTRIBUTED BAYESIAN NETWORK FOR SCHEDULING AND DECISION}

After proposing a distributed BN model Fig.2 and performing parameter learning (using BayesiaLab) to define the probability distribution for every node, this BN is used for scheduling, and also making on-line decision to maintain an acceptable QoC and/or QoS. However, to perform this task, the BN needs information and knowledge about the robot's QoC. A criterion designed to evaluate online QoC is used (Algorithm 1). This algorithm gives the 


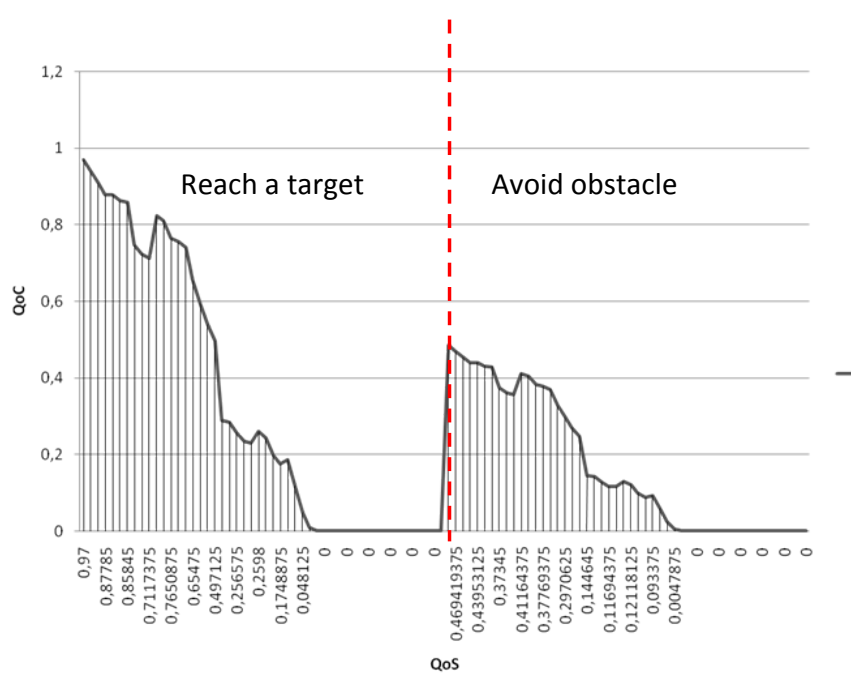

Fig. 3. QoC depending on QoS with two missions: reach a target and avoid abstacle

evidence concerning the QoC of the robot. If the distance $(d)$ between the robot and the station increases, the QoC is considered as bad. Now, if the distance decreases, we verify the orientation error $(\tilde{\theta})$. If the orientation error decreases, then the QoC is good, else the QoC is degraded.

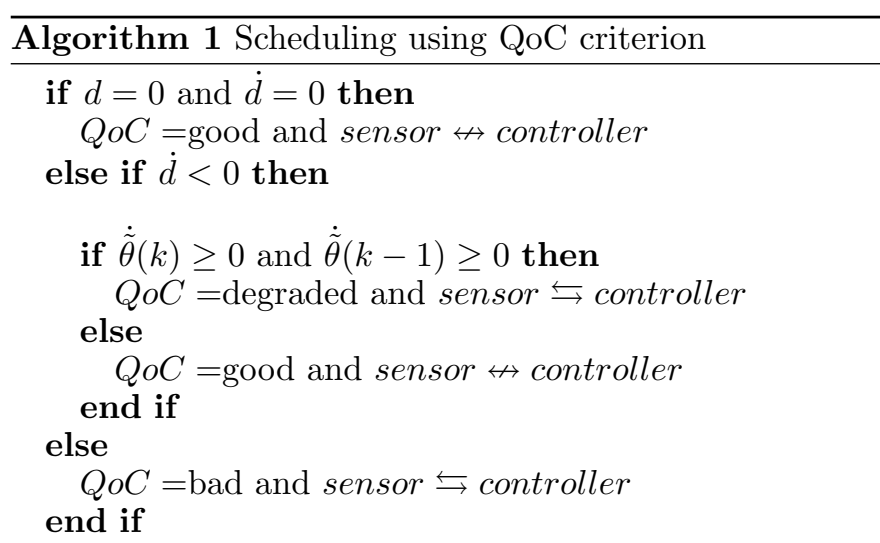

$d$ : distance between the robot and the target, $\tilde{\theta}$ : orientation error.

$\leftrightarrows$ : communication, «: stop communication.

Using information about all the measured variables, the $\mathrm{BN}$ provides a probabilistic estimation of the $\mathrm{QoC}$ and the QoS. Based on this estimation, the robot can make decision to change the scheduling strategy. The objective is to keep the better possible QoC for each robot and the better possible network's QoS.

According to the state of the QoC of the robot (delivered by Algorithm 1) and the state of the QoS (delivered by the $\mathrm{BN}$ knowing the state of the QoC), the system changes the scheduling strategy. Thus, with probability delivered by the BN (Algorithm 2) and Algorithm 1, the values of the conditional probability $P(Q \circ S \mid Q o C, D, C o l, M)$ triggers the sensor task. With only Algorithme 1, the state of the QoC triggers the sensor task of each robot.

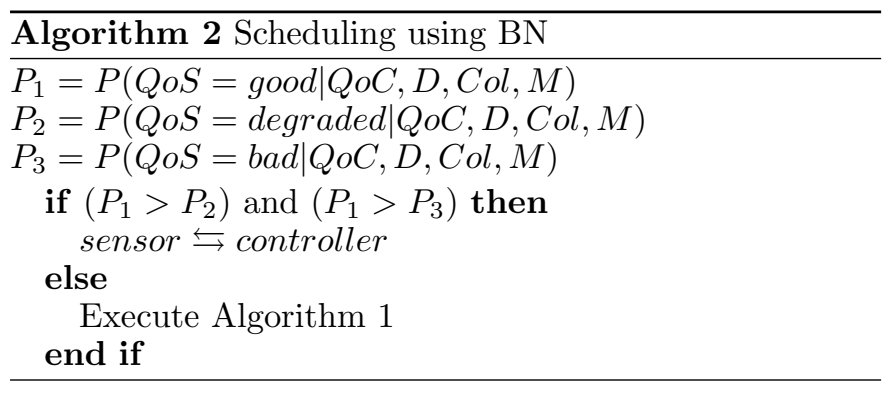

\section{RESULTS}

To verify the efficiency of the proposed BN to schedule and to make decision, two scenarios are presented. In each scenario, the BN and Algorithm 1 are tested and compared with the system without the proposed approach. The use of Algorithm 1 without BN to schedule is also compared with the other strategies (see Table 3 ).

To perform the comparison, five criteria are considered.

(1) The first criterion (Eq. 5) is the Use Request Factor (URF) which represents the frame rate requested to the MAC layer [Juanole and Mouney, 2005]. It influences directly the QoS.

$$
U R F=\sum_{i=1}^{N} \frac{\xi_{i}}{\Gamma * T_{s}^{i}}
$$

$\xi_{i}$ is the length of the frame of task $i . \Gamma$ is the throughput of the WN. $N$ is the number of tasks.

(2) The second criterion concerns also the rate of packet loss. It is calculated as follow:

$$
\Phi=\frac{P_{r}}{P_{s}}
$$

$P_{s}$ is the number of packets sent and $P_{r}$ the number of received packets.

(3) The third criterion concerns the QoC. It is the Integral of the Absolute Error (IAE) (see Eq.7).

$$
I A E=\sum_{k=0}^{p} \epsilon_{x}(k)^{2}+\epsilon_{y}(k)^{2}
$$

$\epsilon_{x}$ and $\epsilon_{y}$ are errors between the reference trajectories (at $0 \%$ of network load) and other trajectory (depending on the network load rate) with respect to $X-$ axis and $Y$ - axis respectively computed with $p$ points representing each trajectory.

(4) The fourth criterion is the fact that the robot reaches the target or not.

(5) The last criterion is the collision between the obstacle and the robot.

\subsection{Scenario 1}

In the first scenario, we suppose that the two robots communicate with one control station. The initial position of the robot 1 is $\left(x_{0}, y_{0}, \theta_{0}\right)=(0,0,0)$ and the position of the target is $\left(x_{d}, y_{d}\right)=(1,1)$. The two robots are always within the coverage area of the station. Hence, the probability $P\left(D<D_{\text {Threshold }}\right)=1$ for each robot.

To limit the resource of the WN and to study the network limits, the throughput is set to 8000bits/s. With this throughput and the sensor tasks of $R 1$ and $R 2$ and the 
controller tasks, the $U R F=64 \%$ in the case of non application of the proposed methods of scheduling (BN and Algorithm1). In this case, the robot reaches the target but it cannot avoid the obstacle. When Algorithm 1 is applied to control the sending of sensor measurements to controllers, it provides the current state of the QoC to the robot. Taking into account this state, a boolean signal for scheduling is generated; "0" starts the communication and "1" stops it. We note that after applying Algorithm 1, URF decreases from $64 \%$ to $34 \%$, the QoS becomes good but the IAE increases from 0.13 to 0.89 for Robot 1 and 0.42 to 1.36 for Robot 2 (see Table 3 ). However, despite the increasing value of IAE, the application of this algorithm has slightly influenced the trajectory and the two robots reach their targets (see Fig. 4) and avoid the obstacle. However, applying the scheduling based on the results of $\mathrm{BN}$, we notice that we have the same performance as with Algorithm 1 except that the IAE drops to zero. This can be explained by the fact that the BN has taken into account the state of the QoS and as it is sufficient to send periodic measurements, the two sensor tasks remain time-triggered. We can conclude that the proposed method is efficient because it takes into account both the QoS and the QoC to make decision and scheduling.

\subsection{Scenario 2}

In this scenario, the same situation as in first scenario is kept but the throughput of the WN is set to 3000bits/s, which means that $U R F=170 \%$ in the case of non application of the proposed methods of scheduling. The IAE is increased from 0.13 to 66.35. Packet losses have increased from 0 to 90 and thus the robot cannot reach the target (Fig. 4) and cannot avoid the obstacle (see Table 3). Now Algorithm 1 is applied to control the sensor task flow. The IAE is decreased from $170 \%$ to $82 \%$. Packet losses are increased but still reasonable. In fact, the robot can reach a target (see Fig. 4) but it cannot avoid the obstacle. Fig. 5 shows the delay with and without the proposed approach. It is clear that the delay with BN is less than the delay without $\mathrm{BN}$. Using results provided from the $\mathrm{BN}$, and with the same scenario, the robot can reach its target (see Fig. 4) and avoid the obstacle. This scenario shows that, the proposed approach is efficient and solves the co-design problem.

\section{CONCLUSION}

In this paper, a novel distributed $\mathrm{BN}$ based approach has been proposed to model the QoS and the QoC of WNCS. This model combines all parameters or variables that influence the Quality of Service of the WN and the Quality of Control of the robot. The BN is designed using statistical learning of experimental data acquired from Khepera III robot. The use of the $\mathrm{BN}$ is justified by the stochastic behavior of the WN. The main objective of the $\mathrm{BN}$ is to make decisions and to control the sensor task flow. According to the situation, the scheduling strategy is applied to guarantee a good QoC of the robot and also to keep a sufficient QoS to send the critical information. The proposed model achieves the desired objectives in terms of scheduling and decision-making. In this paper, the communication architecture for two mobile robots
Table 3. Comparison between the classical approach and the proposed one

\begin{tabular}{|c|c|c|c|c|}
\hline & \multirow{2}{*}{$\begin{array}{c}\text { Scenario1 } \\
64 \%\end{array}$} & \multirow{2}{*}{$\begin{array}{c}\text { Scenario2 } \\
170 \%\end{array}$} \\
\hline \multirow{8}{*}{$\frac{\overline{B N}}{\text { Algo } 1}$} & \multicolumn{2}{|c|}{$\overline{U R F}$} & & \\
\hline & \multirow{3}{*}{$\Phi$} & $R 1$ & $0 \%$ & $91 \%$ \\
\hline & & $R 2$ & $0 \%$ & $96 \%$ \\
\hline & & $S$ & $0 \%$ & $91 \%$ \\
\hline & \multirow[b]{2}{*}{ IAE } & $R 1$ & 0.13 & 66.35 \\
\hline & & $R 2$ & 0.42 & 49.97 \\
\hline & \multicolumn{2}{|c|}{ Reach target } & Yes & No \\
\hline & \multicolumn{2}{|c|}{ Collision } & Yes & Yes \\
\hline \multirow{8}{*}{$\begin{array}{c}\overline{B N} \\
\text { Algo1 }\end{array}$} & \multicolumn{2}{|c|}{$U R F$} & $34 \%$ & $82 \%$ \\
\hline & \multirow{3}{*}{$\Phi$} & $R 1$ & $0 \%$ & $32 \%$ \\
\hline & & $R 2$ & $0 \%$ & $33 \%$ \\
\hline & & $S$ & $0 \%$ & $64 \%$ \\
\hline & \multirow[b]{2}{*}{ IAE } & $R 1$ & 0.89 & 1.31 \\
\hline & & $R 2$ & 1.36 & 1.76 \\
\hline & \multicolumn{2}{|c|}{ Reach target } & Yes & Yes \\
\hline & \multicolumn{2}{|c|}{ Collision } & No & Yes \\
\hline \multirow{8}{*}{$\begin{array}{c}B N \\
\text { Algo1 }\end{array}$} & \multicolumn{2}{|c|}{$U R F$} & $64 \%$ & $79 \%$ \\
\hline & \multirow{3}{*}{$\Phi$} & $R 1$ & $0 \%$ & $33 \%$ \\
\hline & & $R 2$ & $0 \%$ & $30 \%$ \\
\hline & & $S$ & $0 \%$ & $56 \%$ \\
\hline & \multirow[b]{2}{*}{ IAE } & $R 1$ & 0.21 & 0.87 \\
\hline & & $R 2$ & 0.59 & 1.02 \\
\hline & \multicolumn{2}{|c|}{ Reach target } & Yes & Yes \\
\hline & \multicolumn{2}{|c|}{ Collision } & No & No \\
\hline
\end{tabular}

Table 4. Signification of the used abbreviations

\begin{tabular}{lr} 
Abbreviation & Signification \\
\hline $\mathrm{R} 1$ & Robot number 1 \\
$\mathrm{R} 2$ & Robot number 2 \\
$\mathrm{S}$ & Control Station \\
$\Phi$ & Packet loss rate \\
$\mathrm{IAE}$ & Integral of the Absolute Error \\
$\overline{B N}$ & Without BN Approach \\
Algo1 & Without Algorithm 1 \\
Algo1 & With BN approach \\
\hline & With Algorithm 1 \\
\hline
\end{tabular}
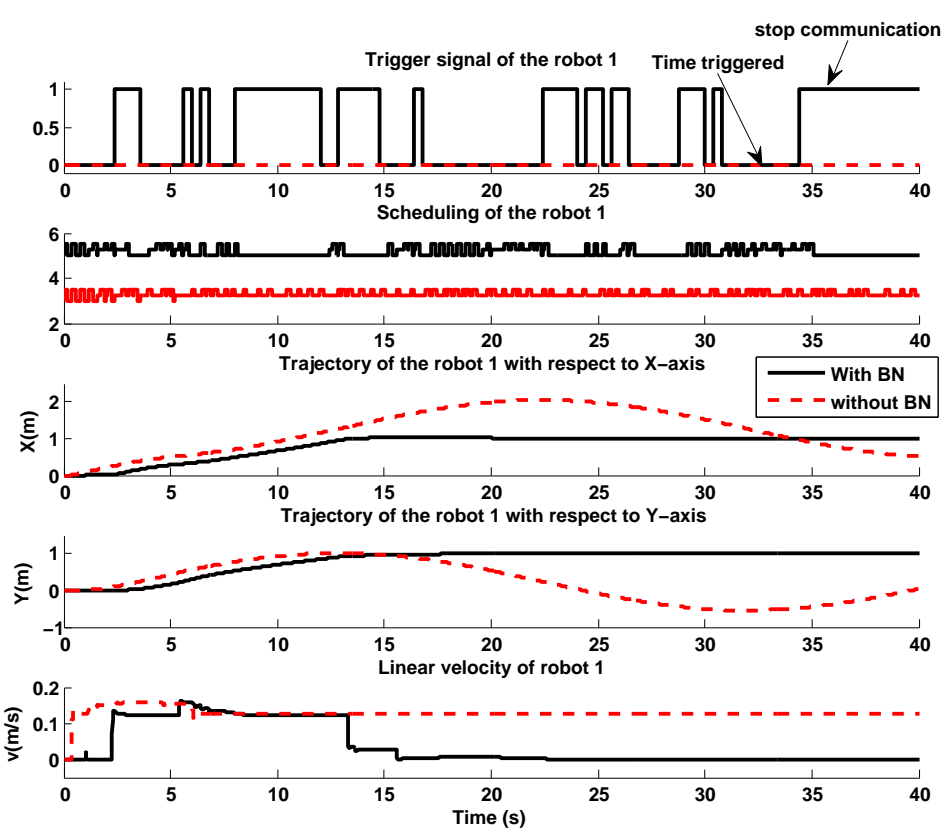

Fig. 4. Comparison between the trajectory of Robot1 with and without BN 


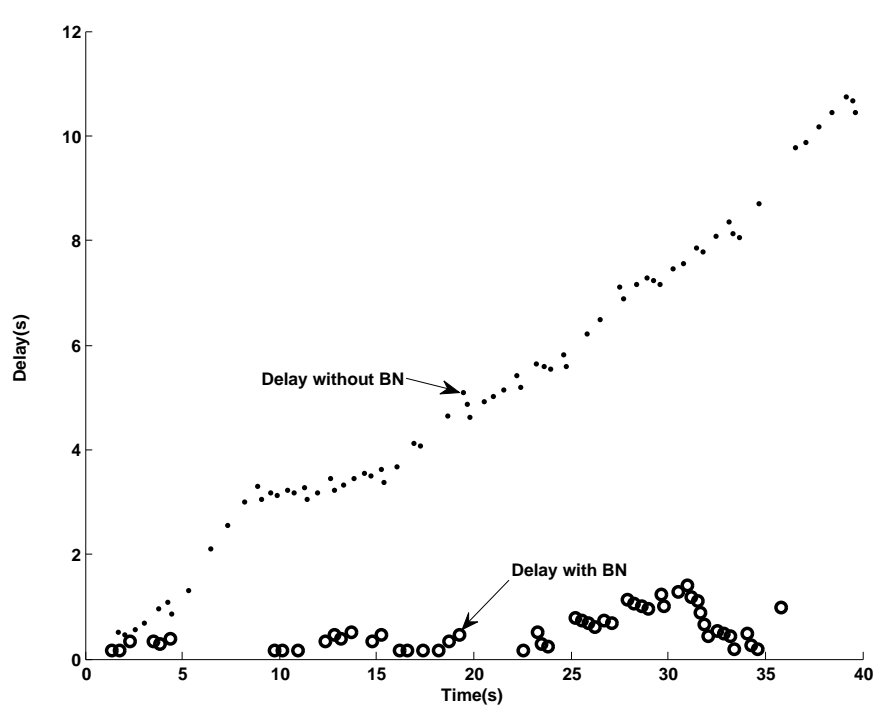

Fig. 5. Comparison between the delay of the sensor task of Robot1 with and without BN

moving in a 2D space with fixed control station has been considered as an illustrative example. According to the information delivered by the proposed BN, the strategy of controlling the sensor flow is applied. It aims to keep a good $\mathrm{QoC}$ in when the robot has to reach a target and avoid obstacle and also to ensure a sufficient QoS. Finally a comparison of the proposed BN scheduling with the classical time-triggered scheduling is performed and the results obtained with $\mathrm{BN}$ scheduling are better in terms of QoS and QoC for WNCS.

In the future work, the degradation of the $\mathrm{QoC}$ and the QoS of WNCS can be avoided by adding some dynamic nodes in two time slices. The probability of the QoC and the QoS in time slice $t+1$ knowing their states in time slice $t$ can help predict the degradation of the QoC.

\section{REFERENCES}

K.-E. Arzen, A. Bicchi, G. Dini, S. Hailes, K. H. Johansson, J. Lygeros, and A. Tzes. A component-based approach to the design of networked control systems. In European Control Conference, Kos , Greece, 2007.

C. Aubrun, D. Simon, and Y.-Q. Song. Co-design Approaches for Dependable Networked Control Systems. Wiley, John and Sons, Incorporated, 2010.

C. Berbra, S. Gentil, and S. Lescecq. Hybrid priority scheme for networked control quadrotor. In $17^{\text {th }}$ Mediterranean Conference on Control and Automation, MED'09, Greece, 2009.

M. S. Branicky, S. M. Phillips, and W. Zhang. Scheduling and feedback co-design for networked control systems. In Proceedings of the $41^{\text {st }}$ IEEE Conference on Decision and Control, Las Vegas, NV, USA, 2002.

M. S. Branicky, V. Liberatore, and S. M. Phillips. Networked control system co-simulation for co-design. In American Control Conference, Denver, USA, 2003.

A. Cervin and J. Eker. Control-scheduling codesign of realtime systems: The control server approach. In Journal of Embedded Computing, volume 1, pages 209-224, 2005.

L. B. Cremean, T. B. Foote, J. H. Gillula, G. H. Hines D. Kogan, K. L. Kriechbaum, J. C. Lamb, J. Leibs L. Lindzey, C. E. Rasmussen, A. D. Stewart, J. W. Bur- dick, and R. M. Murray. Alice: An information-rich autonomous vehicle for high-speed desert navigation. In Journal of Robotic Systems, volume 23, pages 777-810, 2006.

J. Fu and Y. Dai. Mixed $H_{2} / H_{\infty}$ control for networked control systems (NCSs) with markovian packet-loss. In Proceedings of the International Conference on Web Information Systems and Mining, Shanghai, China, pages 563-575, 2009.

D. Giorgiev and D. Tilbury. Packet-based control: The $\mathrm{H}_{2}$-optimal solution. In Automatica, volume 42, pages 137-144, 2006.

Y. Guo and S. Li. A new networked predictive control approach for systems with random network delay in the forward channel. In IEEE Transactions on Industrial Electronics, volume 41, pages 511-520, 2010.

J.P. Hespanha, P. Naghshtabrizi, and Y. Xu. A survey of recent results in networked control systems. In Proceeding of IEEE special issue on Technology of Networked Control Systems, volume 95, pages 138-162, 2007.

G. Juanole and G. Mouney. Qos in real time distributed systems and process control applications. In $1^{\text {st }}$ Workshop on Networked Control Systems and Fault Tolerant Control (NeCST), Ajaccio, France, pages 11-18, 2005.

N. E. Leonard, D. A. Paley, F. Lekien, R. Sepulchre, D. M. Fratantoni, and R. E. Davis. Collective motion, sensor networks, and ocean sampling. In Proceedings of the IEEE, volume 95, pages 48-74, 2007.

L. Li and F.-Y. Wang. Control and communication synthesis in networked control systems. In International Journal of Intelligent Control and Systems, volume 13, pages 81-88, 2008.

F. Liu, Y. Yao, F. He, and S. Chen. Stability analysis of networked control systems with time-varying sampling periods. In Journal of Control Theory and Application, volume 6, pages 22-25, 2008.

A. Mechraoui, Z. H. Khan, J-M. Thiriet, and S. Gentil. Codesign for wireless networked control of an intelligent mobile robot. In International Conference on Informatics in Control, Automation and Robotics (ICINCO), Milan, Italy, 2009.

A. Mechraoui, J.-M. Thiriet, and S. Gentil. On-line distributed bayesian decision and diagnosis of wireless networked mobile robots. In $18^{\text {th }}$ Mediterranean Conference on Control and Automation, Marrakech, Maroc, 2010.

P. Naim, P.H. Wuillemin, P. Leray, O. Pourret, and A. Becker. Rseaux baysiens. Eyrolles, $3^{\text {rd }}$ edition, 2004.

J. Pearl. Probabilistic reasoning in intelligent systems: Networks of plausible inference. Morgan Kaufman Publishers, 1988.

B. Sinopoli, L. Schenato, M. Franceschetti, K. Poolla, M. Jordan, and S. Sastry. Kalman filtering with intermittent observations. In IEEE Transactions on Automatic Control, volume 49, pages 1453-1464, 2004.

D. H. Varsakelis and L. Zhang. LQG control of networked control systems with access constraints and delays. In International Journal of Control, volume 81, pages 1266-1280, 2008.

Z. Xiaomei, Z. Yufan, and L. Guoping. Stochastic stability of networked control systems with network-induced delay and data dropout. In Journal of control theory and applications, volume 6, pages 405-409, 2008. 